\title{
Observations on macrolide resistance and susceptibility testing performance in field isolates collected from clinical bovine respiratory disease cases
}

\author{
Keith D DeDonder ${ }^{\mathrm{a}^{*}}$, Dayna M Harhay ${ }^{\mathrm{b}}$, Michael D Apley ${ }^{\mathrm{c}}$, Brian V Lubbers ${ }^{\mathrm{d}}$, Michael L \\ Clawson ${ }^{\mathrm{b}}$, Gennie Schuller ${ }^{\mathrm{b}}$, Gregory P Harhay ${ }^{\mathrm{b}}$, Brad J White ${ }^{\mathrm{c}}$, Robert L Larson ${ }^{\mathrm{c}}$, Sarah F \\ Capik $^{\mathrm{a}}$, Jim E Riviere ${ }^{\mathrm{e}}$, Ted Kalbfleisch ${ }^{\mathrm{f}}$, Ronald K Tessman ${ }^{\mathrm{g}}$
}

${ }^{a}$ Diagnostic Medicine/Pathobiology, Kansas State University College of Veterinary Medicine, Manhattan, KS, United States;

${ }^{\mathrm{b} U S D A}$ ARS US Meat Animal Research Center, Clay Center, NE, United States;

${ }^{c}$ Clinical Sciences, Kansas State University College of Veterinary Medicine, Manhattan, KS, United States;

${ }^{\mathrm{d}}$ Kansas State Veterinary Diagnostic Laboratory, Kansas State University College of Veterinary Medicine, Manhattan, KS, United States;

${ }^{\mathrm{e}}$ Institute of Computational Comparative Medicine, Kansas State University College of Veterinary Medicine, Manhattan, KS, United States;

${ }^{\mathrm{f}}$ Biochemistry and Molecular Genetics Department, School of Medicine, University of Louisville, Louisville, KY, United States;

${ }^{g}$ Pharmaceutical Research and Development, Merial, Duluth, GA, United States.

Running title: bovine respiratory disease and macrolide resistance

*Corresponding author. Tel: +1-785-320-2369; E-mail: keith@mvsinc.net; Present address: Veterinary and Biomedical Research Center, Inc., Manhattan, KS, United States

\section{Page 1 of 26}

(C) 2016. This manuscript version is made available under the Elsevier user license http://www.elsevier.com/open-access/userlicense/1.0/ 


\begin{abstract}
The objectives of this study were; first, to describe gamithromycin susceptibility of Mannheimia haemolytica, Pasteurella multocida, and Histophilus somni isolated from cattle diagnosed with bovine respiratory disease (BRD) and previously treated with either gamithromycin for control of BRD (mass medication=MM) or sham-saline injected (control=CON); second, to describe the macrolide resistance genes present in genetically typed M. haemolytica isolates; third, use whole-genome sequencing (WGS) to correlate the phenotypic resistance and genetic determinants for resistance among $M$. haemolytica isolates. $M$. haemolytica $(\mathrm{n}=276)$, P. multocida $(\mathrm{n}=253)$, and H. somni $(\mathrm{n}=78)$ were isolated from feedlot cattle diagnosed with BRD. Gamithromycin susceptibility was determined by broth microdilution. Whole-genome sequencing was utilized to determine the presence/absence of macrolide resistance genes and to genetically type $M$. haemolytica. Generalized linear mixed models were built for analysis. There was not a significant difference between MM and CON groups in regards to the likelihood of culturing a resistant isolate of $M$. haemolytica or $P$. multocida. The likelihood of culturing a resistant isolate of $M$. haemolytica differed significantly by state of origin in this study. A single $M$. haemolytica genetic subtype was associated with an over whelming majority of the observed resistance. H. somni isolation counts were low and statistical models would not converge. Phenotypic resistance was predicted with high sensitivity and specificity by WGS. Additional studies to elucidate the relationships between phenotypic expression of resistance/genetic determinants for resistance and clinical response to antimicrobials are necessary to inform judicious use of antimicrobials in the context of relieving animal disease and suffering.
\end{abstract}


Key words: gamithromycin; macrolide; antimicrobial resistance; bovine respiratory disease; Mannheimia haemolytica; Pasteurella multocida; Histophilus somni

\section{Introduction}

Antimicrobial administration has been the mainstay for both the treatment and control of bovine respiratory disease (BRD) for several decades. Considering the ability of bacteria to adapt and survive in changing environmental conditions, it comes as no surprise that the bacterial pathogens associated with BRD are now commonly found to be resistant to these antimicrobials (DeDonder and Apley, 2015).

One of the first reports on macrolide resistance genes found in BRD was an investigation of a strain of Pasteurella multocida (strain 36950) isolated from a calf in a Nebraska feedlot in 2005 (Kadlec et al., 2011). This particular strain had shown resistance to most antimicrobials approved for treatment of BRD and prior to their investigation, the mechanism of resistance had not been determined. Performing whole genome sequencing (WGS) and a series of cloning experiments they discovered three macrolide resistance genes, (the first of which was described in a nearly simultaneous publication (Desmolaize et al., 2011a)) $\operatorname{erm}(42), m s r(\mathrm{E})$, and $m p h(\mathrm{E})$. The erm(42) gene codes for an rRNA methylase gene, the $m s r(\mathrm{E})$ gene codes for an $\mathrm{ABC}$ transporter protein, and the $m p h(\mathrm{E})$ gene codes for a macrolide phosphotransferase protein (Kadlec et al., 2011; Michael et al., 2012b). The $m s r(\mathrm{E})$ and $m p h(\mathrm{E})$ genes are organized in an operon-like structure and separated only by a non-coding spacer sequence of 55 base pairs.

A recent study investigated the effects of these three genes on the minimal inhibitory concentration (MIC) of gamithromycin and tildipirosin (Michael et al., 2012a). In both $P$. multocida and Mannheimia haemolytica they reported a pronounced increase in MIC to 
gamithromycin for those isolates containing $m \operatorname{sr}(\mathrm{E})-m p h(\mathrm{E})$ and a marked increase in MIC to tildipirosin for erm(42) containing isolates. The subset of isolates that harbored all three genes displayed increased MICs to both of the newer macrolides in their study. Similar work found the same to be true; greatly elevated MIC for tildipirosin and tilmicosin in the presence of erm(42) and greatly elevated MIC for tilmicosin, tulathromycin, and gamithromycin in the presence of $m s r(\mathrm{E})-m p h(\mathrm{E})$ (Rose et al., 2012). These two studies remain the only publications to investigate the molecular effects of the macrolide resistance genes on BRD bacterial pathogen susceptibility to gamithromycin. However, neither investigated the effects of a macrolide antimicrobial administered for control of BRD on subsequent macrolide resistance.

Therefore, the objectives of this work were: first, to describe gamithromycin susceptibility of M. haemolytica, P. multocida, and Histophilus somni isolated from cattle diagnosed with and treated for BRD, which had previously either received treatment for control of BRD (MM) or sham-saline injection $(\mathrm{CON})$, and second, to characterize the macrolide resistance genes present in the genetically typed $M$. haemolytica isolates. The final objective was to use whole-genome sequencing (WGS) to describe genetic determinants for macrolide resistance and correlate to the phenotypic resistance as determined by broth microdilution among the M. haemolytica isolates from this BRD clinical trial.

\section{Materials and methods}

\subsection{Animals and treatment allocation}

The randomized clinical trial yielding the isolates described in this manuscript was detailed previously in a pharmacokinetic/pharmacodynamic investigation which utilized the same isolates (DeDonder et al., 2015). One hundred eighty cattle perceived to be at a high risk for developing BRD (165-269 kgs) were sourced from three states (Kentucky, Missouri, and 
Tennessee; 60 head per state) within the United States as part of another study. The cattle were randomly allocated to receive either treatment for control of BRD (mass medication $=\mathrm{MM})$ with gamithromycin $(6 \mathrm{mg} / \mathrm{kg}$ subcutaneously in the neck) or a sham injection of saline (control $=$ CON) administered upon arrival to a research feedlot near Manhattan, Kansas. The treatments were assigned to cattle within state of source, yielding a total of six pens ( 30 head/pen) in the study. This study was approved by the Kansas State University Institutional Animal Care and Use Committee.

\subsection{Clinical scoring and disease diagnosis}

Cattle were observed for symptoms of BRD once daily in their pen for 28 days by a veterinarian masked to treatment allocation. The diagnosis of BRD was determined based on a calf having a rectal temperature of $\geq 40.0^{\circ} \mathrm{C}\left(\geq 104.0^{\circ} \mathrm{F}\right)$ and a clinical score of $\geq 1$ (general signs

of depression and weakness). Cattle diagnosed with BRD based on this clinical scoring system were included in this study and administered gamithromycin at the label dose after the collection of pre-treatment samples. Therefore, cattle enrolled (treated for BRD) in this study had been previously administered either MM (gamithromycin for control of BRD) or CON (sham control injection) on arrival to the feeding facility.

\subsection{Sampling allocation and collection procedures}

Nasopharyngeal swabs (NPS) were collected, bilaterally and pooled, immediately prior to treatment (0 hour) from all cattle diagnosed with BRD. Additionally, cattle were allocated to having bronchoalveolar lavage (BAL) collection at either 0,12 , or 24 hours post-treatment with gamithromycin. Cattle sampled by BAL at 12 and 24 hours also were simultaneously sampled by NPS. All cattle diagnosed with BRD were sampled by means of both BAL and NPS 120 hours after treatment administration. 


\subsection{Bacterial isolation and MIC determination}

After collection, NPS and BAL samples were transported on ice and were plated directly onto trypticase soy $+5 \%$ blood, chocolate, and MacConkey agar plates. The plates were incubated in $5 \% \mathrm{CO}_{2}$ at $37^{\circ} \mathrm{C}$ for $18-24$ hours. Up to 12 colonies of $M$. haemolytica and up to 6 colonies of both $P$. multocida and $H$. somni displaying growth characteristics typical of each were further isolated in pure culture from each calf. Isolate identity was confirmed with MALDITOF (Bruker Daltonics, Billerica, MA, USA) and the selected isolates were frozen for subsequent susceptibility testing.

Gamithromycin susceptibility testing of all isolates was determined by a broth microdilution technique utilizing custom frozen panels (CML1FZAC plates (TREK Diagnostic Systems, Thermo-Fisher Scientific Inc., Waltham, MA, USA)), as per manufacturers' instructions, by personnel masked to treatment group according to CLSI recommended techniques (Clinical and Laboratory Standards Institute, 2013). The frozen panels contained gamithromycin at concentrations ranging from 0.03 to $16 \mathrm{mg} / \mathrm{L}$. Prior to susceptibility testing, isolates were recovered from frozen stock by culturing on Chocolate II agar plates (Becton Dickinson, Co. Sparks, MD) and incubation with increased $\mathrm{CO}_{2}$ at $37^{\circ} \mathrm{C}$ for $18-20$ hours. Suspensions of M. haemolytica and P. multocida, equivalent to $0.5 \mathrm{McFarland}$ standard, were made in $5 \mathrm{~mL}$ of demineralized water. Mueller-Hinton broth tubes $(11 \mathrm{~mL})$ were then inoculated with $140 \mu \mathrm{L}$ of the resulting bacterial suspensions. A 12-channel pipette was used to dispense 50 $\mu \mathrm{L}$ of this suspension into each of 12 wells in the plate, such that the susceptibility of 8 strains could be evaluated per plate. Plates were sealed with seal strips and incubated at $37^{\circ} \mathrm{C}$ without $\mathrm{CO}_{2}$ for 18-20 hours. H. somni isolates were tested as per CLSI guideline VET01-A4, section 12.2 (Clinical and Laboratory Standards Institute, 2013). In brief, H. somni cultures were 
cultivated on Chocolate II agar plates in $5 \% \pm 2 \% \mathrm{CO}_{2}$, at $37^{\circ} \mathrm{C}$, for $24 \mathrm{~h}$. Resulting colonies were picked to $5 \mathrm{ml}$ Mueller-Hinton broth tubes (TREK Diagnostic Systems), to achieve an optical density equivalent to 0.5 McFarland standard. This suspension was used to inoculate $2 \mathrm{X}$ Veterinary Fastidious Medium (VFM; TREK Diagnostic Systems) at a ratio of $15 \mathrm{ul}$ per $1 \mathrm{ml}$, and the resulting bacterial suspension was dispensed $50 \mathrm{ul}$ per well. Plates were sealed prior to incubation in $5 \% \mathrm{CO}_{2}$ at $37^{\circ} \mathrm{C}$ for $24 \mathrm{~h}$. For all strains tested, growth was visually inspected and Gamithromycin MIC determined as the lowest concentration preventing visible growth.

Interpretation of susceptible, intermediate or resistant was determined using clinical breakpoints established by CLSI. Gamithromycin breakpoints have been reported for $M$. haemolytica, $P$. multocida, and $H$. somni at $\leq 4,8, \geq 16 \mathrm{mg} / \mathrm{L}$ for susceptible, intermediate, and resistant, respectively (Clinical and Laboratory Standards Institute, 2015). Staphylococcus aureus ATCC 29213 was used to evaluate quality control of the single batch CML1FZAC plates $(\mathrm{n}=100)$ used in this study. Plates were tested at three time points (beginning, mid-point and end) with MIC values found within QC range throughout the study.

\subsection{M. haemolytica genomic sequencing, bioinformatic, and phylogenetic analyses}

The details of these methods have been previously reported (Capik et al., 2016). Briefly, extracted DNA was quantified and Illumina Nextera XT DNA libraries were constructed and sequenced (minimum 10X sequence reads per genome) on a DNA sequencer (Illumina MiSeq DNA Sequencer, San Diego, CA, USA). Resulting library sequences were individually mapped to an available closed circular M. haemolytica genome available in GenBank (CP004752) (Harhay et al., 2013). Bootstrapped Neighbor-Joining phylogenetic trees were constructed from SNP genotypes that were identified from the mapped sequences. Additionally, antimicrobial resistance genes were identified by mapping to previously described integrative and conjugative 
elements (ICE) identified in two M. haemolytica isolates (GenBank \# CP004752 and CP005383.1) (Harhay et al., 2013), and one P. multocida (GenBank \# NC_016808) (Eidam et al., 2015) isolate.

\subsection{Statistical analysis}

Intermediate and susceptible isolates of each bacterial species were grouped together to create a binomial outcome variable (either susceptible/intermediate or resistant) for purposes of analysis. Data were inputted into a statistical software (STATA/SE 12.1 for Windows; StataCorp LP, College Station, TX, USA) and generalized linear mixed models, fit by maximum-likelihood regression, were built to determine if there was an association between on-arrival treatment and an MIC determination of susceptible/intermediate or resistant. Treatment group, sample time point, sample source and state of origin were modeled as fixed effects and random variables were included to account for clustering of isolates within cattle and cattle within individual pens for the analysis of $M$. haemolytica data. Models including state of origin would not converge for $P$. multocida data. Neither models would converge when a treatment by sampling time point interaction was included. All attempts to model H. somni data were unsuccessful due to a low isolation count. A corresponding $P$-value of $\leq 0.05$ was considered significant.

\subsection{Comparing M. haemolytica isolate gamithromycin MICs to the presence or absence of} macrolide resistance genes erm(42), $\operatorname{msr}(E)-m p h(E)$

In order to determine if WGS resistance determinants correctly predict the phenotypic resistance (susceptible/intermediate or resistant) for $M$. haemolytica a two by two table was constructed. This was used to calculate MIC test accuracy, sensitivity, specificity, and positive and negative predictive values as compared to the presence or absence of the macrolide resistance genes. For the purposes of this report a True Positive was assumed to be an isolate 
containing resistance genes and determined as resistant by MIC testing. Likewise, a True Negative was assumed to be an isolate not containing macrolide resistance genes and determined as susceptible/intermediate by MIC testing. The accuracy for this study was calculated as (True Positive + True Negative) / Total of Isolates. The sensitivity in this study was defined as the proportion of the isolates that contained the $\operatorname{erm}(42), m s r(\mathrm{E})$, and $m p h(\mathrm{E})$ genes that were identified as resistant by MIC determination and was calculated as True Negative / (False Positive + True Negative). The specificity was defined as the proportion of isolates that lacked the $\operatorname{erm}(42), \operatorname{msr}(\mathrm{E})$, and $m p h(\mathrm{E})$ genes with MICs below the susceptible breakpoint and was calculated as True Negative / (False Positive + True Negative). The positive predictive value for resistance was defined as the probability that an isolate which contained the $\operatorname{erm}(42), m s r(\mathrm{E})$, and $m p h(\mathrm{E})$ genes was determined resistant by MIC testing and was calculated as True Positive / (True Positive + False Positive). The negative predictive value for susceptibility was defined as the probability that an isolate lacking the $\operatorname{erm}(42), \operatorname{ms}(\mathrm{E})$, and $m p h(\mathrm{E})$ genes was determined by MIC testing determined to be susceptible and was calculated as True Negative / (False Negative + True Negative). There were no steps to account for multiple isolates per calf in this portion of the analysis as the MIC testing was performed at the individual isolate level.

\section{Results}

\subsection{Morbidity}

As previously reported, 26 cattle met the clinical criteria for BRD during the course of the study (DeDonder et al., 2015). There were only four first-treatment failures, two each from the MM and CON groups. The number of days on study at the time of BRD diagnosis ranged from $4-21$ days, with a mean and median of 14 days. Of the four treatment failures, two yielded 
a $M$. haemolytica isolate over the sampling period and both of those calves harbored $M$. haemolytica strains containing resistant genes at the final sampling.

\subsection{MIC distribution of M. haemolytica, P. multocida, and H. somni}

The MIC distributions of M. haemolytica $(\mathrm{n}=287)$, P. multocida $(\mathrm{n}=257)$, and H. somni $(\mathrm{n}=78)$ are presented in Table 1, segregated by treatment administered upon feedlot arrival and further by whether they were isolated pre- or post-treatment for BRD with gamithromycin. The MICs of the M. haemolytica and P. multocida isolates were previously described in a separate investigation of the pharmacokinetics/pharmacodynamics of gamithromycin (DeDonder et al., 2015).

\subsection{Macrolide resistance gene presence by M. haemolytica subtype}

There were 287 isolates of M. haemolytica collected throughout this study by both NPS and BAL. Those 287 isolates were successfully cultured from just 14 of the 26 cattle (53.8\%) diagnosed with BRD. Eleven of those 14 cattle were from the CON group and the remaining

three cattle were from the MM group. The range of isolates from both sample sources (24 maximum possible isolates) per calf per time point was from $0-24$ isolates with a median of 6 isolates/calf. Genetic subtype data could not be retrieved for eleven of the 287 isolates, leaving 276 isolates of M. haemolytica for analysis. The 276 isolates of M. haemolytica subjected to WGS fell into one of two distinct phylogenetic clades and each clade was further divided into subtypes based on clustering patterns and strong bootstrap support (Capik et al., 2016).

Thirty nine isolates of $M$. haemolytica were isolated over all time points from the nine head of cattle in the MM group and only one isolate (2c subtype) displayed resistance to gamithromycin. This isolate was from a calf originating from Missouri which was collected 120 
hours after treatment for BRD. Further, the MM cattle had only a single isolate in the $2 \mathrm{~b}$ subtype. This isolate was cultured prior to treatment and categorized as susceptible with an MIC of 1 $\mathrm{mg} / \mathrm{L}$, and was negative for the both the $\operatorname{erm}(42)$ gene and the $m s r(\mathrm{E})-m p h(\mathrm{E})$ operon.

Eighty-six of the 89 (96.6\%) post-treatment isolates in the CON group categorized as phenotypically resistant came from the $2 \mathrm{~b}$ subtype and all of these resistant $2 \mathrm{~b}$ isolates came from the same pen of cattle, from just four calves all originating from Kentucky. The three isolates not from the $2 \mathrm{~b}$ group which displayed phenotypic resistance in CON cattle were isolated from cattle originating from the states of Missouri (1f), Tennessee (1i), and Kentucky (2d), respectively. Just a single isolate, $M$. haemolytica subtype $2 \mathrm{c}$, displayed phenotypic resistance in MM cattle.

Although there are numerical differences in the counts of resistant $M$. haemolytica between the MM and CON treatment groups, there was no effect of treatment (MM or CON) observed on the likelihood of isolating a resistant M. haemolytica $(P=0.93)$. There was, however, an observed effect of sampling time point on the likelihood of isolating resistant M. haemolytica $(P<0.0001)$. Samples obtained from either NPS or BAL at 120 hours after treatment administration were more likely to contain resistant isolates as compared to those obtained at time $0(\mathrm{OR}=395,95 \%$ CI 23-6,733; $\mathrm{P}<0.001)$. Additionally, samples obtained at 12 hours posttreatment administration were also significantly different than those obtained prior to treatment $(\mathrm{OR}=67,95 \%$ CI 1.2-3,730; $P=0.04)$ in regards to the likelihood of culturing resistant isolate of M. haemolytica.

There was a significant effect of state of origin $(P=0.04)$ on the likelihood of culturing a resistant isolate of $M$. haemolytica. Calves originating from Kentucky were significantly more 
likely $(\mathrm{OR}=514,95 \% \mathrm{CI} 2.7-98,117 ; P=0.02)$ to yield a resistant isolate as compared to calves from Missouri and Tennessee.

No statistical differences were found between treatment groups in regards to the likelihood of isolating a resistant $P$. multocida $(P=0.98)$ and there was no observed effect of sampling time point $(P=0.32)$. Models in which state of origin was included did not converge for P. multocida. Due to the low isolation counts, all attempts at models for $H$. somni were unsuccessful.

All M. haemolytica isolates collected in this study were negative for the presence of the $\operatorname{erm}(42)$ gene. The only isolates in which the $\operatorname{msr}(\mathrm{E})-m p h(\mathrm{E})$ operon were detected were in the $2 \mathrm{~b}$ subtype; all of the cattle yielding these isolates originated from Kentucky and the isolates displayed phenotypic resistance of MIC $\geq 16 \mathrm{mg} / \mathrm{L}$. All $2 \mathrm{~b}$ isolates collected prior to treatment had an MIC of $1 \mathrm{mg} / \mathrm{L}$ and were negative for the $m s r(\mathrm{E})-m p h(\mathrm{E})$ operon. Eighty-five of 88 $(96.6 \%)$ of the $2 \mathrm{~b}$ subtype isolates collected after treatment for BRD with gamithromycin contained the $m s r(\mathrm{E})-m p h(\mathrm{E})$ operon. In all cases the $m s r(\mathrm{E})-m p h(\mathrm{E})$ was part of an ICE, however, there were other isolates that contained an ICE but didn't harbor the $m s r(\mathrm{E})-m p h(\mathrm{E})$ operon (data not shown).

\subsection{Association between gene presence and MIC}

Minimal inhibitory concentration by genetic subtype can be found in Table 2. Five of the clade 1 subtypes had MIC determination spread across the susceptible, intermediate, or resistant categories (1f pre-treatment, and $1 \mathrm{i}, 2 \mathrm{~b}, 2 \mathrm{c}$, and $2 \mathrm{~d}$ post-treatment). Comparison of the phenotype to the genotype was performed for all $276 \mathrm{M}$. haemolytica isolates. There were 16 discrepancies (16 individual isolates from 5 cattle) between phenotypic MIC determination and genotype and all were isolates collected post-treatment. The animal identification number, sample source 
(BAL or NPS), treatment group, $M$. haemolytica subtype, MIC determination, and status of the $m s r(\mathrm{E})-m p h(\mathrm{E})$ for each of the isolates that had a mismatch of phenotype and genotype can be found in Table 3. Interestingly, each of the isolates which displayed phenotypic resistance that were not from the $2 \mathrm{~b}$ subtype (a single isolate from each of subtype $1 \mathrm{f}, 1 \mathrm{i}, 2 \mathrm{c}$, and $2 \mathrm{~d}$ ) reverted to a phenotype of susceptible (MIC=1 mg/L, data not shown) on subsequent MIC testing as part of another experiment. The repeated testing (isolates frozen between testing from late Fall of 2013 to early spring of 2015) for that experiment was targeted at the isolates with an MIC of $16 \mathrm{mg} / \mathrm{L}$. However, given that the initial call was resistant with no identifiable laboratory errors, and that the retesting was biased and un-blinded, and not all isolates could feasibly be retested, the intial calls were not changed.

The isolates in 1f and $1 \mathrm{i}$ were predominately susceptible with an MIC value of 1 , the one isolate from each of these two subtypes that resulted in an MIC of $\geq 16 \mathrm{mg} / \mathrm{L}$ appeared to be either misclassified (due to the absence of detectable $m s r(\mathrm{E})-m p h(\mathrm{E})$ genes) by MIC testing or displayed phenotypic resistance through another mechanism. Post-treatment isolates of subtype $2 \mathrm{~b}$ ( $\mathrm{n}=88$ from 4 calves) resulted in 86 resistant $(\geq 16 \mathrm{mg} / \mathrm{L})$ and two susceptible $(1 \mathrm{mg} / \mathrm{L}) \mathrm{MIC}$ determinations. The two susceptible $2 \mathrm{~b}$ isolates did not contain the $m s r(\mathrm{E})-m p h(\mathrm{E})$ operon and both originated from one calf which also yielded 17 other $2 \mathrm{~b}$ isolates containing the $m s r(\mathrm{E})$ $m p h(\mathrm{E})$ operon.

The two by two table populated with the M. haemolytica isolate results from this study can be found in Table 4. Additionally in Table 4 are the calculated sensitivity, specificity, accuracy, positive predictive value, and negative predictive values. 


\section{Discussion}

In the study reported here, the observed effect on gamithromycin susceptibility, after administering gamithromycin for the control of BRD on arrival versus sham-saline injected controls was not as expected. When accounting for clustering of cattle within pen and isolates within cattle there was not a statistical difference between treatment groups $(P=0.93)$. In contrast, there was a numerically higher number of strains displaying resistance in both $P$. multocida and $H$. somni in the cattle that received MM on arrival to the feedlot. There was not a statistical difference between treatment groups $(\mathrm{P}=0.98)$ for $P$. multocida detected in this study. However, in this naturally occurring BRD study the sample size was potentially insufficient to detect a difference if one truly exists in regard to the difference in phenotypic resistance induced between MM and CON cattle. Statistical models for H. somni would not converge due to limited sample size.

There was a significant difference by state of origin in the likelihood of obtaining a resistant isolate of $M$. haemolytica. Cattle originating from Kentucky were significantly more likely to yield a resistant isolate as compared to cattle sourced from either Missouri or Tennessee. Reasons for the geographical trend observed in this study are not clear but could include different management practices or antimicrobial usage patterns prior to the cattle being marketed and subsequently included in this study, or it is possible that real geographical differences in the prevalence of resistance could exist. However, it should be pointed out that these results are from a large number of isolates originating from a small sample size of cattle and further research in this area is warranted.

Comparing only the MIC distributions of the isolates collected post-treatment between

the MM and CON cattle (Table 1); treatment for BRD appeared to increase the prevalence of 
phenotypic resistance in $M$. haemolytica isolated from CON cattle, and increase the prevalence of phenotypic resistance in P. multocida and H. somni isolated from MM cattle. Additionally, while all 26 cattle had NPS pre-treatment, only eight BAL samples were collected pre-treatment and 44 (nine each at 12 and $24 \mathrm{hrs,} \mathrm{and} 26$ at $120 \mathrm{hrs)} \mathrm{post-treatment.} \mathrm{These} \mathrm{factors} \mathrm{could}$ possibly confound the results in this study since there were more opportunities for isolation of resistant (or susceptible) bacteria across these three post-treatment time points. However, it is interesting to note that the observed increase of phenotypic resistance in P. multocida and $H$. somni did not result in numerous overt clinical failures. This is further evidence of a possible disconnect between in vitro testing results and treatment outcome, especially as it pertains to the macrolide class of antimicrobials, as has been previously reported (Amsden, 2001; Kanoh and Rubin, 2010; McClary et al., 2011).

A single M. haemolytica subtype (2b) represented all genotypic macrolide resistance and 95.6\% (86/90) of phenotypic resistance. The $2 \mathrm{~b}$ isolates came from just five calves and two of these five calves ultimately ended up as clinical failures. Nearly ninety-nine percent (89/90) of the phenotypic resistance in $M$. haemolytica came from CON cattle. Just one single strain, of the 2c subtype, phenotypically expressed resistance among the isolates from the MM cattle and this strain did not harbor the $\operatorname{erm}(42)$ or $m s r(\mathrm{E})-m p h(\mathrm{E})$ macrolide resistance genes, so alternative genomic resistance factors should be considered.

There is no clear explanation as to the over-representation of $M$. haemolytica resistance observed in the CON cattle. Perhaps there is a synergism between gamithromycin and the immune system (or other factors) when administered as a MM on arrival (i.e. to a non-clinical animal) that is not present when a calf becomes morbid with symptoms of BRD. Macrolides are known to possess anti-inflammatory and immunomodulatory effects in addition to their 
antimicrobial activity (Amsden, 2005; Kanoh and Rubin, 2010). While no such data are available on gamithromycin, another veterinary macrolide has been extensively studied in this area (Duquette et al., 2015; Er and Yazar, 2012; Fischer et al., 2013; Fischer et al., 2011; Fischer et al., 2014). Additionally, azithromycin, a macrolide of the same subclass as gamithromycin, has been shown to exert anti-inflammatory properties on lung epithelial cells in humans (Kitsiouli et al., 2015). Researchers have noted that the administration of azithromycin to healthy human volunteers resulted in acute stimulation of neutrophil degranulation and phagocytosis-associated oxidative burst together with increases in serum interleukin-1 $\beta$ which the authors stated may contribute towards its antimicrobial activity (Culic et al., 2002).

A further intriguing observation from this study is the inability to find M. haemolytica isolates containing $\operatorname{erm}(42)$ or $\operatorname{msr}(\mathrm{E})-\operatorname{mph}(\mathrm{E})$ macrolide resistance genes in any samples cultured prior to treatment for BRD (Table 2), especially considering that nine of the 26 cattle had previously received a macrolide class antimicrobial as a treatment for control of BRD. One conventional theory is that bacteria may contain the genetic machinery for resistance but not express it phenotypically until after selection pressure is applied (antimicrobial administration), allowing the proliferation of the resistant bacterial strains. However, we observed no detectable macrolide resistance genes in pre-treatment isolates (from both MM and CON cattle). There was one isolate (subtype 1f) that was classified as resistant but the macrolide resistance genes $\operatorname{erm}(42), \operatorname{msr}(\mathrm{E})$, and $m p h(\mathrm{E})$ were not detected. Yet in isolates of M. haemolytica that were collected after the antimicrobial was administered for treatment of BRD, we observed the presence of the $m s r(\mathrm{E})-m p h(\mathrm{E})$ operon in 85 of $88(96.6 \%)$ of the $2 \mathrm{~b}$ subtype. It remains unclear as to why just one particular subtype contained the macrolide resistance genes and all of the others did not. 
This finding could support multiple hypotheses. It is possible that a small population of M. haemolytica strains were present containing the macrolide resistance genotype that were not successfully cultured because they are present below a minimum level of detection, or the strains harboring the resistance genes were present in refugia, within biofilms or as persister cells, or present in an area of the respiratory tract not sampled. Additionally, it is possible that there were no $M$. haemolytica strains present containing the resistance genes prior to treatment and they were quickly acquired by horizontal transfer from other bacterial cells such as $P$. multocida, as described by Michael et al. (Michael et al., 2012c).

One recent study reported finding $\operatorname{erm}(42)$ and $\operatorname{msr}(\mathrm{E})-m p h(\mathrm{E})$, being carried in $M$. haemolytica isolates independent from one another very infrequently (Michael et al., 2012a). In this study reported here, no isolates contained the $\operatorname{erm}(42)$ gene, however, the $m s r(\mathrm{E})-m p h(\mathrm{E})$ genes were found frequently in the $2 \mathrm{~b}$ subtype of $M$. haemolytica isolates. Although the maximum antimicrobial test concentration was $16 \mathrm{mg} / \mathrm{L}$ for MIC testing of gamithromycin, this study demonstrated that the presence of $m s r(\mathrm{E})-m p h(\mathrm{E})$ alone can increase the MIC of $M$. haemolytica to gamithromycin from 1 to $\geq 16 \mathrm{mg} / \mathrm{L}$ for the $2 \mathrm{~b}$ subtype. Other researchers have also observed dramatic increases in MICs for isolates harboring the macrolide resistance genes $\operatorname{erm}(42)$ and $m s r(\mathrm{E})-m p h(\mathrm{E})$ for erythromycin, tilmicosin, and tulathromycin (Desmolaize et al., 2011a; Kadlec et al., 2011; Klima et al., 2014). While MIC data for these other macrolides were not determined in the study reported here, a similar increase in MICs to each of those antimicrobials for the isolates containing the macrolide resistance genes $m s r(\mathrm{E})-m p h(\mathrm{E})$ would be expected. Further, given prior evidence of macrolide cross-resistance (Desmolaize et al., 2011b; Kadlec et al., 2011; Van Donkersgoed et al., 2008; Watts et al., 1994) and the fact that resistance to tulathromycin was observed in BRD isolates prior to it being licensed for use 
(Portis et al., 2012) also substantiate an expectation of macrolide cross-resistance in the isolates from this study.

Overall a very high sensitivity (100\%), specificity (97.4\%), and accuracy (98.2\%) was observed in the MIC determination of gamithromycin susceptibility for the M. haemolytica that were isolated in this study. Additionally, the positive predictive value and negative predictive values were both high at $94 \%$ and $100 \%$, respectively. Positive and negative predictive values are dependent upon the prevalence of resistance in the dataset and can be limited when resistance is not present in the isolates tested (Klement et al., 2005). Resistant strains were well represented in this study; therefore, these values can be considered as reliable indicators of the probability of the MIC determination of susceptibility to gamithromycin by broth microdilution technique being truly correct for $M$. haemolytica isolates in populations of cattle that are similar to those in this study.

There are several possible reasons for the discrepancy observed in the $2 \mathrm{~d}$ isolates and the other (1f, 1i, 2b, 2c) discrepant results in this study. First, it is possible that another mechanism of resistance, such as a de novo mutation, was present in some of the isolates in this subtype which was undetected by our techniques. In a recent report, one M. haemolytica (strain Mh14717) and three P. multocida (strains Pm14424, Pm14421, Pm14426) that were confirmed negative for the $\operatorname{erm}(42)$ and $\operatorname{msr}(\mathrm{E})-m p h(\mathrm{E})$ displayed high macrolide resistance (Olsen et al., 2015). Those isolates were from the field and collected from cattle within Europe from 2010 2013. The methodology used in that investigation utilized DNA extraction and Multiplex PCR to confirm the absence of previously reported macrolide resistance genes, and subsequent genome sequencing to attempt to determine the mechanism of resistance. In all strains they reported that the observed resistance was conferred by $23 \mathrm{~S}$ rRNA mutations and speculated that it was from 
de novo mutations followed by selective pressure (administration of macrolide antimicrobial). The isolates in the present study that were resistant and intermediate to gamithromycin but did not contain the erm(42) and $m s r(\mathrm{E})-m p h(\mathrm{E})$ genes were investigated for mutations (post hoc) in 23S rRNA and no such mutations were found. It is possible that there are other factors or resistant elements not accounted for in this study that are responsible for the observed increase in MIC within these strains.

Another possible explanation is regarding the fact that the MIC determination and DNA sequencing were performed independently at different points in time. Although both diagnostics utilized the same bacterial stock, it is possible that a mixed culture of two different subtypes of M. haemolytica was present in the stock cultures.

A final hypothesis for the discrepancy between phenotype and genotype could be due to inherent variability in MIC determination. Even under ideal conditions MIC testing is reported to be subject to variability from sources such as inoculum, consistency of medium composition, incubation temperature and atmosphere, reading of the test, among others (Frimodt-Moller, 2002; Mitchell et al., 2012). Although a high degree of repeatability was observed in this current study and the QC strain remained within the QC range throughout, these limitations could help explain the variability that was observed, especially considering the sensitivity in MIC determinations that macrolides can display with even slight changes in $\mathrm{pH}$ and media composition (Evans, 2005; Godinho et al., 2005; Mitchell et al., 2013; Mitchell et al., 2012).

In a study similar to the one reported here, a high correlation between resistance genotype and resistance phenotype was observed (Tyson et al., 2015). A macrolide similar to gamithromycin, azithromycin, was included in their testing panel and they reported a $100 \%$ sensitivity and specificity. However, they observed very low resistance rates, with only one of 76 
strains of E. coli containing resistance genes to azithromycin. Although the organism in their study was $E$. coli and the antimicrobial panel was more extensive, we too report a similarly high correlation between antimicrobial resistance genotype and phenotypic expression in $M$. haemolytica for the macrolide gamithromycin. Other groups have reported similar high concordance between genotype and phenotype in human (Gordon et al., 2014; Stoesser et al., 2013) and porcine (Zankari et al., 2013) isolates using WGS.

\section{Conclusions}

The objectives of this publication were to describe gamithromycin susceptibility of $M$. haemolytica, P. multocida, and H. somni isolated from cattle diagnosed with BRD, and second, to characterize the macrolide resistance genes present in genetically subtyped $M$. haemolytica isolated from these same cattle, and third to use WGS to correlate the phenotypic resistance and genetic determinants for resistance among the $M$. haemolytica isolates. The results indicate a very complex, and perhaps bacterial species dependent, interaction is taking place between the host, the bacterial pathogen, and the antimicrobial. Further, this interaction appears to be confounded by the state from which the cattle originate. The geographical trend observed in this study deserves further attention to determine what factors might cause such a difference.

The M. haemolytica subtype $2 \mathrm{~b}$ was the only strain to display genotypic resistance to gamithromycin in this study. Despite the findings of genotypic resistance, a high likelihood of first-treatment success was observed following administration of gamithromycin for the treatment of BRD and overt clinical failure did not seem to be associated with the macrolide resistance genes identified. 
Future studies aimed at further investigating the prevalence and incidence of resistance and its correlation with $M$. haemolytica subtype could lead to the development of a specific vaccine if a suitable epitope was discovered. If an efficacious vaccine could be developed against a strain of $M$. haemolytica that is predisposed for antimicrobial resistance it would be a major advancement in the area of BRD prevention and therapy.

Additional studies to elucidate the relationships between phenotypic expression of resistance/genetic determinants for resistance and clinical response to antimicrobials are necessary to inform judicious use of antimicrobials in the context of relieving animal disease and suffering.

In this study, WGS was utilized to determine the presence or absence of the macrolide resistance genes $\operatorname{erm}(42)$ and $\operatorname{msr}(\mathrm{E})-m p h(\mathrm{E})$ and to correlate those results with the gamithromycin susceptibility pattern observed. These data demonstrate that WGS may be effectively used to determine the presence or absence of the resistant genes, and at some point in the future could serve as a reasonable alternative to phenotypic testing procedures and lead to quick and rapid detections assays, like polymerase chain reaction (PCR) assays.

\section{Acknowledgments}

The authors wish to thank Max Andersen, Kelly Lechtenberg, Sara McReynolds, Kelsey McClure, Bradley Robért, and Mal Hoover for their assistance with this project. The mention of trade names or commercial products in this article is solely for the purpose of providing specific information and does not imply recommendation or endorsement by the U.S. Department of Agriculture. The USDA is an equal opportunity employer. 


\section{Conflict of interest statement}

R.K.T. was an employee of Merial at the time of this research. Other authors: none to declare.

\section{Funding}

This study was financially supported, in part, by Merial, Duluth, Georgia, United States. 


\section{References}

Amsden, G.W., 2001. Advanced-generation macrolides: tissue-directed antibiotics. Int J Antimicrob Agents 18 Suppl 1, S11-15.

Amsden, G.W., 2005. Anti-inflammatory effects of macrolides--an underappreciated benefit in the treatment of community-acquired respiratory tract infections and chronic inflammatory pulmonary conditions? J Antimicrob Chemother 55, 10-21.

Capik, S.F., White, B.J., Lubbers, B.V., Apley, M.D., DeDonder, K.D., Larson, R.L., Harhay, G.P., Chitko-McKown, C.G., Harhay, D.M., Kalbfleisch, T., Schuller, G., Clawson, M.L., 2016. Comparison of the diagnostic performance of nasopharyngeal swabs and bronchoalveolar lavages in calves diagnosed with bovine respiratory disease Am J Vet Res In press.

Clinical and Laboratory Standards Institute, 2013. Performance Standards for Antimicrobial Disk and Dilution Susceptibility Tests fro Bacteria Isolated From Animals; Approved Standard- 4th Edition. CLSI document VET01-A4. .

Clinical and Laboratory Standards Institute, 2015. Performance Standards for Antimicrobial Disk and Dilution Susceptibility Tests for Bacteria isolated from Animals; Approved Standard - 3rd Edition CLSI Supplement VET01S.

Culic, O., Erakovic, V., Cepelak, I., Barisic, K., Brajsa, K., Ferencic, Z., Galovic, R., Glojnaric, I., Manojlovic, Z., Munic, V., Novak-Mircetic, R., Pavicic-Beljak, V., Sucic, M., Veljaca, M., Zanic-Grubisic, T., Parnham, M.J., 2002. Azithromycin modulates neutrophil function and circulating inflammatory mediators in healthy human subjects. Eur $\mathbf{J}$ Pharmacol 450, 277-289.

DeDonder, K.D., Apley, M.D., 2015. A literature review of antimicrobial resistance in Pathogens associated with bovine respiratory disease. Anim Health Res Rev 16, 125-134.

DeDonder, K.D., Apley, M.D., Li, M., Gehring, R., Harhay, D.M., Lubbers, B.V., White, B.J., Capik, S.F., KuKanich, B., Riviere, J.E., Tessman, R.K., 2015. Pharmacokinetics and pharmacodynamics of gamithromycin in pulmonary epithelial lining fluid in naturally occurring bovine respiratory disease in multi-source commingled feedlot cattle. J Vet Pharmacol Ther

Desmolaize, B., Rose, S., Warrass, R., Douthwaite, S., 2011a. A novel Erm monomethyltransferase in antibiotic-resistant isolates of Mannheimia haemolytica and Pasteurella multocida. Mol Microbiol 80, 184-194.

Desmolaize, B., Rose, S., Wilhelm, C., Warrass, R., Douthwaite, S., 2011b. Combinations of macrolide resistance determinants in field isolates of Mannheimia haemolytica and Pasteurella multocida. Antimicrob Agents Chemother 55, 4128-4133.

Duquette, S.C., Fischer, C.D., Williams, A.C., Sajedy, S., Feener, T.D., Bhargava, A., Reti, K.L., Muench, G.P., Morck, D.W., Allison, J., Lucas, M.J., Buret, A.G., 2015. Immunomodulatory effects of tulathromycin on apoptosis, efferocytosis, and proinflammatory leukotriene B4 production in leukocytes from Actinobacillus pleuropneumoniae-or zymosan-challenged pigs. Am J Vet Res 76, 507-519.

Eidam, C., Poehlein, A., Leimbach, A., Michael, G.B., Kadlec, K., Liesegang, H., Daniel, R., Sweeney, M.T., Murray, R.W., Watts, J.L., Schwarz, S., 2015. Analysis and comparative genomics of ICEMh1, a novel integrative and conjugative element (ICE) of Mannheimia haemolytica. J Antimicrob Chemother 70, 93-97. 
Er, A., Yazar, E., 2012. Effects of tylosin, tilmicosin and tulathromycin on inflammatory mediators in bronchoalveolar lavage fluid of lipopolysaccharide-induced lung injury. Acta Vet Hung 60, 465-476.

Evans, N.A., 2005. Tulathromycin: an overview of a new triamilide antibiotic for livestock respiratory disease. Vet Ther 6, 83-95.

Fischer, C.D., Beatty, J.K., Duquette, S.C., Morck, D.W., Lucas, M.J., Buret, A.G., 2013. Direct and indirect anti-inflammatory effects of tulathromycin in bovine macrophages: inhibition of CXCL-8 secretion, induction of apoptosis, and promotion of efferocytosis. Antimicrob Agents Chemother 57, 1385-1393.

Fischer, C.D., Beatty, J.K., Zvaigzne, C.G., Morck, D.W., Lucas, M.J., Buret, A.G., 2011. AntiInflammatory benefits of antibiotic-induced neutrophil apoptosis: tulathromycin induces caspase-3-dependent neutrophil programmed cell death and inhibits NF-kappaB signaling and CXCL8 transcription. Antimicrob Agents Chemother 55, 338-348.

Fischer, C.D., Duquette, S.C., Renaux, B.S., Feener, T.D., Morck, D.W., Hollenberg, M.D., Lucas, M.J., Buret, A.G., 2014. Tulathromycin exerts proresolving effects in bovine neutrophils by inhibiting phospholipases and altering leukotriene B4, prostaglandin E2, and lipoxin A4 production. Antimicrob Agents Chemother 58, 4298-4307.

Frimodt-Moller, N., 2002. How predictive is PK/PD for antibacterial agents? Int J Antimicrob Agents 19, 333-339.

Godinho, K.S., Keane, S.G., Nanjiani, I.A., Benchaoui, H.A., Sunderland, S.J., Jones, M.A., Weatherley, A.J., Gootz, T.D., Rowan, T.G., 2005. Minimum inhibitory concentrations of tulathromycin against respiratory bacterial pathogens isolated from clinical cases in European cattle and swine and variability arising from changes in in vitro methodology. Vet Ther 6, 113-121.

Gordon, N.C., Price, J.R., Cole, K., Everitt, R., Morgan, M., Finney, J., Kearns, A.M., Pichon, B., Young, B., Wilson, D.J., Llewelyn, M.J., Paul, J., Peto, T.E., Crook, D.W., Walker, A.S., Golubchik, T., 2014. Prediction of Staphylococcus aureus antimicrobial resistance by whole-genome sequencing. J Clin Microbiol 52, 1182-1191.

Harhay, G.P., Koren, S., Phillippy, A.M., McVey, D.S., Kuszak, J., Clawson, M.L., Harhay, D.M., Heaton, M.P., Chitko-McKown, C.G., Smith, T.P., 2013. Complete Closed Genome Sequences of Mannheimia haemolytica Serotypes A1 and A6, Isolated from Cattle. Genome Announc 1.

Kadlec, K., Brenner Michael, G., Sweeney, M.T., Brzuszkiewicz, E., Liesegang, H., Daniel, R., Watts, J.L., Schwarz, S., 2011. Molecular basis of macrolide, triamilide, and lincosamide resistance in Pasteurella multocida from bovine respiratory disease. Antimicrob Agents Chemother 55, 2475-2477.

Kanoh, S., Rubin, B.K., 2010. Mechanisms of action and clinical application of macrolides as immunomodulatory medications. Clin Microbiol Rev 23, 590-615.

Kitsiouli, E., Antoniou, G., Gotzou, H., Karagiannopoulos, M., Basagiannis, D., Christoforidis, S., Nakos, G., Lekka, M.E., 2015. Effect of azithromycin on the LPS-induced production and secretion of phospholipase A2 in lung cells. Biochim Biophys Acta 1852, 1288-1297.

Klement, E., Chaffer, M., Leitner, G., Shwimmer, A., Friedman, S., Saran, A., Shpigel, N., 2005. Assessment of accuracy of disk diffusion tests for the determination of antimicrobial susceptibility of common bovine mastitis pathogens: a novel approach. Microb Drug Resist 11, 342-350. 
Klima, C.L., Zaheer, R., Cook, S.R., Booker, C.W., Hendrick, S., Alexander, T.W., McAllister, T.A., 2014. Pathogens of bovine respiratory disease in North American feedlots conferring multidrug resistance via integrative conjugative elements. J Clin Microbiol 52, 438-448.

McClary, D.G., Loneragan, G.H., Shryock, T.R., Carter, B.L., Guthrie, C.A., Corbin, M.J., Mechor, G.D., 2011. Relationship of in vitro minimum inhibitory concentrations of tilmicosin against Mannheimia haemolytica and Pasteurella multocida and in vivo tilmicosin treatment outcome among calves with signs of bovine respiratory disease. $\mathrm{J}$ Am Vet Med Assoc 239, 129-135.

Michael, G.B., Eidam, C., Kadlec, K., Meyer, K., Sweeney, M.T., Murray, R.W., Watts, J.L., Schwarz, S., 2012a. Increased MICs of gamithromycin and tildipirosin in the presence of the genes erm(42) and msr(E)-mph(E) for bovine Pasteurella multocida and Mannheimia haemolytica. J Antimicrob Chemother 67, 1555-1557.

Michael, G.B., Kadlec, K., Sweeney, M.T., Brzuszkiewicz, E., Liesegang, H., Daniel, R., Murray, R.W., Watts, J.L., Schwarz, S., 2012b. ICEPmu1, an integrative conjugative element (ICE) of Pasteurella multocida: analysis of the regions that comprise 12 antimicrobial resistance genes. J Antimicrob Chemother 67, 84-90.

Michael, G.B., Kadlec, K., Sweeney, M.T., Brzuszkiewicz, E., Liesegang, H., Daniel, R., Murray, R.W., Watts, J.L., Schwarz, S., 2012c. ICEPmu1, an integrative conjugative element (ICE) of Pasteurella multocida: structure and transfer. J Antimicrob Chemother 67, 91-100.

Mitchell, J.D., Goh, S., McKellar, Q.A., McKeever, D.J., 2013. In vitro pharmacodynamics of gamithromycin against Mycoplasma mycoides subspecies mycoides Small Colony. Vet J (London, England : 1997) 197, 806-811.

Mitchell, J.D., McKellar, Q.A., McKeever, D.J., 2012. Pharmacodynamics of antimicrobials against Mycoplasma mycoides mycoides small colony, the causative agent of contagious bovine pleuropneumonia. PloS One 7, e44158.

Olsen, A.S., Warrass, R., Douthwaite, S., 2015. Macrolide resistance conferred by rRNA mutations in field isolates of Mannheimia haemolytica and Pasteurella multocida. $\mathrm{J}$ Antimicrob Chemother 70, 420-423.

Portis, E., Lindeman, C., Johansen, L., Stoltman, G., 2012. A ten-year (2000-2009) study of antimicrobial susceptibility of bacteria that cause bovine respiratory disease complex-Mannheimia haemolytica, Pasteurella multocida, and Histophilus somni--in the United States and Canada. J Vet Diagn Invest 24, 932-944.

Rose, S., Desmolaize, B., Jaju, P., Wilhelm, C., Warrass, R., Douthwaite, S., 2012. Multiplex PCR to identify macrolide resistance determinants in Mannheimia haemolytica and Pasteurella multocida. Antimicrob Agents Chemother 56, 3664-3669.

Stoesser, N., Batty, E.M., Eyre, D.W., Morgan, M., Wyllie, D.H., Del Ojo Elias, C., Johnson, J.R., Walker, A.S., Peto, T.E., Crook, D.W., 2013. Predicting antimicrobial susceptibilities for Escherichia coli and Klebsiella pneumoniae isolates using whole genomic sequence data. J Antimicrob Chemother 68, 2234-2244.

Tyson, G.H., McDermott, P.F., Li, C., Chen, Y., Tadesse, D.A., Mukherjee, S., Bodeis-Jones, S., Kabera, C., Gaines, S.A., Loneragan, G.H., Edrington, T.S., Torrence, M., Harhay, D.M., Zhao, S., 2015. WGS accurately predicts antimicrobial resistance in Escherichia coli. J Antimicrob Chemother 70, 2763-2769. 
Van Donkersgoed, J., Merrill, J., Hendrick, S., 2008. Comparative efficacy of tilmicosin versus tulathromycin as a metaphylactic antimicrobial in feedlot calves at moderate risk for respiratory disease. Vet Ther 9, 291-297.

Watts, J.L., Yancey, R.J., Jr., Salmon, S.A., Case, C.A., 1994. A 4-year survey of antimicrobial susceptibility trends for isolates from cattle with bovine respiratory disease in North America. J Clin Microbiol 32, 725-731.

Zankari, E., Hasman, H., Kaas, R.S., Seyfarth, A.M., Agerso, Y., Lund, O., Larsen, M.V., Aarestrup, F.M., 2013. Genotyping using whole-genome sequencing is a realistic alternative to surveillance based on phenotypic antimicrobial susceptibility testing. $\mathbf{J}$ Antimicrob Chemother 68, 771-777. 
Table 1 - MIC to gamithromycin of $M$. haemolytica $(\mathrm{n}=276), P$. multocida $(\mathrm{n}=253)$, and $H$. somni $(\mathrm{n}=78)$ isolated from cattle $(n=26)$ diagnosed with bovine respiratory disease prior to treatment (Pre-Tx) for BRD and a summation of isolates collected at 12,24 , and 120 hours following treatment (Post-Tx) with gamithromycin.

\begin{tabular}{|c|c|c|c|c|c|c|c|c|c|c|c|c|}
\hline & & & & & nimur & Inhit & ory ( & nce & tic & ng & & \\
\hline & & & $\leq 0.03$ & 0.06 & 0.12 & 0.25 & 0.5 & 1 & 2 & 4 & 8 & $\geq 16$ \\
\hline & & Pre-Tx & - & - & - & - & - & 89 & 2 & - & - & 1 \\
\hline & 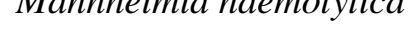 & Post-Tx & - & - & - & - & - & 46 & - & - & 11 & 88 \\
\hline 롤 & & Pre-Tx & - & - & - & 1 & 33 & 17 & - & - & - & 1 \\
\hline ฮี II & Pasteurella multocida & Post-Tx & - & - & - & - & 19 & 8 & - & - & - & 2 \\
\hline & & Pre-Tx & - & 3 & 6 & 37 & 1 & 1 & - & - & - & 1 \\
\hline & Histophilus somni & Post-Tx & - & - & - & - & - & - & - & - & - & - \\
\hline & & Pre-Tx & - & - & - & - & - & 26 & - & - & - & - \\
\hline & Mannheımıa haemolytıca & Post-Tx & - & - & - & - & - & 10 & 1 & 1 & - & 1 \\
\hline है & Pasteurella multocida & Pre-Tx & - & - & - & - & - & - & - & - & 1 & 49 \\
\hline$\Sigma \Sigma^{\prime \prime}$ & Pasteurella multocida & Post-Tx & - & - & - & - & 1 & - & - & - & - & 121 \\
\hline & & Pre-Tx & - & - & - & - & - & - & - & - & - & 6 \\
\hline & Histophilus somni & Post-Tx & - & - & - & - & - & - & - & - & - & 23 \\
\hline
\end{tabular}


Table 2-MIC to gamithromycin of M. haemolytica isolates $(\mathrm{n}=276)$ from either the control group $(\mathrm{CON})$ or those cattle that were mass medicated (MM) with gamithromycin on arrival.

\begin{tabular}{|c|c|c|c|c|c|c|c|c|c|c|c|c|}
\hline \multirow[b]{2}{*}{ Treatment } & \multirow[b]{2}{*}{ Subtype } & & \multicolumn{10}{|c|}{ Minimum Inhibitory Concentration $(\mathrm{mg} / \mathrm{L})$} \\
\hline & & $\begin{array}{l}\text { Time } \\
\text { Point }\end{array}$ & 0.03 & 0.06 & 0.12 & 0.25 & 0.5 & 1 & 2 & 4 & 8 & $\geq 16$ \\
\hline \multirow[t]{10}{*}{$C O N$} & \multirow[t]{2}{*}{$1 \mathrm{e}$} & Pre-Tx & - & - & - & - & - & 2 & 2 & - & - & - \\
\hline & & Post-Tx & - & - & - & - & - & - & - & - & - & - \\
\hline & \multirow[t]{2}{*}{$1 \mathrm{f}$} & Pre-Tx & - & - & - & - & - & 11 & - & - & - & 1 \\
\hline & & Post-Tx & - & - & - & - & - & 19 & - & - & - & - \\
\hline & \multirow[t]{2}{*}{$1 \mathrm{i}$} & Pre-Tx & - & - & - & - & - & 12 & - & - & - & - \\
\hline & & Post-Tx & - & - & - & - & - & 19 & - & - & - & 1 \\
\hline & \multirow[t]{2}{*}{$2 b$} & Pre-Tx & - & - & - & - & - & 42 & - & - & - & - \\
\hline & & Post-Tx & - & - & - & - & - & 2 & - & - & - & $86^{\wedge}$ \\
\hline & \multirow[t]{2}{*}{$2 d$} & Pre-Tx & - & - & - & - & - & 22 & - & - & - & - \\
\hline & & Post-Tx & - & - & - & - & - & 6 & - & - & 11 & 1 \\
\hline \multirow[t]{6}{*}{$M M$} & \multirow[t]{2}{*}{$1 \mathrm{c}$} & Pre-Tx & - & - & - & - & - & 1 & - & - & - & - \\
\hline & & Post-Tx & - & - & - & - & - & - & - & - & - & - \\
\hline & \multirow[t]{2}{*}{$2 b$} & Pre-Tx & - & - & - & - & - & 1 & - & - & - & - \\
\hline & & Post-Tx & - & - & - & - & - & - & - & - & - & - \\
\hline & \multirow[t]{2}{*}{$2 \mathrm{c}$} & Pre-Tx & - & - & - & - & - & 24 & - & - & - & - \\
\hline & & Post-Tx & - & - & - & - & - & 10 & 1 & 1 & - & 1 \\
\hline
\end{tabular}

$*$ Pre-Tx $=$ Pre-treatment; Post-Tx $=$ Summation of isolates collected at 12, 24, and 120 hours following treatment $\mathrm{CON}=$ control treatment group; $\mathrm{MM}=$ mass medication with gamithromycin

${ }^{\wedge}$ One isolate with $\mathrm{MIC} \geq 16 \mathrm{mg} / \mathrm{L}$ did not contain detectable $m s r(\mathrm{E})-\operatorname{mph}(\mathrm{E})$ macrolide resistance genes 
Table 3 - Sample source, treatment group, M. haemolytica subtype, MIC determination and status of $m s r(\mathrm{E})-m p h(\mathrm{E})$ resistance genes for each of the isolates whose genotype did not match their phenotype.*

\begin{tabular}{|c|c|c|c|c|c|}
\hline Animal ID & $\begin{array}{l}\text { Sample } \\
\text { Source }\end{array}$ & Treatment & Subtype & MIC & $m s r(E)-m p h(E)$ \\
\hline 104 & NPS & $\mathrm{CON}$ & 1f & $\geq 16$ & $\mathrm{~N}$ \\
\hline 175 & NPS & $\mathrm{CON}$ & $1 \mathrm{i}$ & $\geq 16$ & $\mathrm{~N}$ \\
\hline 222 & NPS & $\mathrm{CON}$ & $2 b$ & $\geq 16$ & $\mathrm{~N}$ \\
\hline 206 & NPS & MM & $2 c$ & $\geq 16$ & $\mathrm{~N}$ \\
\hline \multirow{12}{*}{243} & BAL & $\mathrm{CON}$ & $2 d$ & $\geq 16$ & $\mathrm{~N}$ \\
\hline & BAL & $\mathrm{CON}$ & $2 d$ & 8 & $\mathrm{~N}$ \\
\hline & BAL & $\mathrm{CON}$ & $2 d$ & 8 & $\mathrm{~N}$ \\
\hline & BAL & $\mathrm{CON}$ & $2 d$ & 8 & $\mathrm{~N}$ \\
\hline & BAL & $\mathrm{CON}$ & $2 d$ & 8 & $\mathrm{~N}$ \\
\hline & BAL & $\mathrm{CON}$ & $2 d$ & 8 & $\mathrm{~N}$ \\
\hline & BAL & $\mathrm{CON}$ & $2 \mathrm{~d}$ & 8 & $\mathrm{~N}$ \\
\hline & BAL & $\mathrm{CON}$ & $2 \mathrm{~d}$ & 8 & $\mathrm{~N}$ \\
\hline & BAL & $\mathrm{CON}$ & $2 d$ & 8 & $\mathrm{~N}$ \\
\hline & BAL & $\mathrm{CON}$ & $2 d$ & 8 & $\mathrm{~N}$ \\
\hline & BAL & $\mathrm{CON}$ & $2 d$ & 8 & $\mathrm{~N}$ \\
\hline & NPS & $\mathrm{CON}$ & $2 d$ & 8 & $\mathrm{~N}$ \\
\hline
\end{tabular}

*NPS = nasopharyngeal swab, BAL = bronchoalveolar lavage fluid; $\mathrm{CON}=$ control treatment group; $\mathrm{MM}=$ mass medication with gamithromycin upon feedlot arrival 
Table 4 - Two by two table of genotype, as determined by presence/absence of $m s r(\mathrm{E})-m p h(\mathrm{E})$, versus phenotype, as determined by broth microdilution, test outcome for the M. haemolytica isolates in this study.*

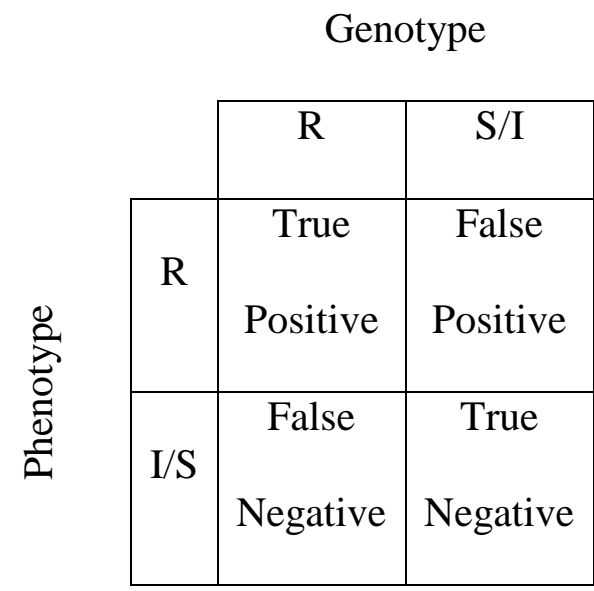

\begin{tabular}{|c|c|c|c|}
\hline & \multicolumn{2}{|c|}{ Genotype } \\
\hline & & $\mathrm{R}$ & $\mathrm{S} / \mathrm{I}$ \\
\hline \multirow{2}{*}{ 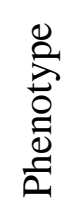 } & $\mathrm{R}$ & 85 & 5 \\
\hline & $\mathrm{I} / \mathrm{S}$ & 0 & 186 \\
\hline
\end{tabular}

Sensitivity

$100 \%$

Specificity $97.4 \%$

Accuracy

$98.2 \%$

Positive Predictive Value

$94 \%$

Negative Predictive Value

$100 \%$

$* R=$ Resistant $;$ I/S = Intermediate/Susceptible 